Rev. Saúde públ., S. Paulo, 20(1):83-101, 1986

\title{
ASPECTOS SÓCIO-ECONOMICOS DA DESNUTRIÇÃO NO BRASIL
}

\author{
Antonio Carlos Coelho Campino*
}

CAMPINO, A.C.C. Aspectos sócio-econômicos da desnutrição no Brasil. Rev. Saúde públ., S. Paulo,20:83-101, 1986.

RESUMO: Objetiva-se mostrar os fatores sócio-econômicos que têm sido identificados como os principais determinantes da situação nutricional de um país. Concluise que a renda é o fator isoladamente mais importante na determinação do estado nutricional, mas uma vez fixada esta variável, outros fatores - tais como extensão do sistema de atendimento de saúde, nível educacional, programa de alimentação também desempenham um papel relevante. Procura-se avaliar, empiricamente, com base em pesquisas até agora realizadas, quais seriam os determinantes da situação nutricional para o Brasil. Evidencia-se a hipótese, esperada na literatura, de que a renda é o fator mais importante, e, dado esta, também no caso brasileiro surgem como fatores relevantes o acesso a serviços de saúde e saneamento. Em face disto, discutem-se algumas alternativas de uma política de nutrição, mostrando-se a magnitude da redistribuição de renda necessária para cobrir o hiato nutricional e debatendo-se o papel dos programas de alimentação e nutrição, na forma em que foram explicitados no Programa de Prioridades Sociais do atual Governo.

UNITERMOS: Desnutrição. Fatores sócio-econômicos, Renda. População. Criança, estado nutricional.

\section{1 - INTRODUÇÃO}

É sabido que uma parcela significativa da população brasileira padece de deficiência nutricional. Estimativa recente indica que 45 milhões de pessoas apresentariam déficit calórico superior a $20 \%$ das necessidades (Campino e Farina, ${ }^{4}$ 1985).

A identificação das eventuais medidas de política, que permitiriam reduzir a intensidade da deficiência nutricional e a magnitude da população afetada pela mesma, depende do conhecimento dos fatores sócio-econômicos que têm sido identificados como os principais determinantes da situação nutricional.

Este trabalho visa, portanto, identificar os principais determinantes da situação nutricional de um país. Concluindose que a renda é o fator isoladamente mais importante na determinação do estado nutricional, e uma vez fixada esta variável, outros fatores como a extensão do sistema de saúde, nível educacional e a existência de programas de alinnentação também desempenham um papel importante. Em seguida, procurou-se avaliar empiricamente os fatores associados à situação nutricional e de consumo de alimentos no Brasil, evidenciando-se as mesmas hipóteses gerais esperadas na literatura.

A parte final deste artigo destina-se a uma digressão acerca de uma política de nutrição para o Brasil. Discutem-se as limitações de uma política redistributiva e debate-se o papel dos programas de alimentação e nutrição na forma em que foram explicitados no Programa de Prioridades Sociais do atual Governo.

\section{2 - DETERMINANTES SÓCIO-ECONÔMI- COS DO ESTADO NUTRICIONAL}

Um esquema de análise das inter-relações entre o estado nutricional de uma população em geral e de crianças em particular é apresentado por Levinson ${ }^{15}$ (1974) e reproduzido a seguir:

\footnotetext{
* Da Faculdade de Economia e Administracão da Universidade de São Paulo - Av. Prof. Luciano Gualberto, 908 - Cidade Universitária - 05508 - São Paulo, SP - Brasil.
} 
CAMPINO, A.C.C. Aspectos sócio-econômicos da desnutrição no Brasil. Rev. Saúde públ., S. Paulo, 20:83-101, 1986.

Determinantes do Estado Nutricional

- Conteúdo nutricional dos alimentos

- Presença ou ausência de programas de alimentação fora do domicílio

- Poder de compra da família (ren-? da e preços)

- Crenças maternas sobre nutrição e saúde

- Extensão do Sistema de Atendi...:- Presença ou ausência Ingestão de alimentos mento de Saúde

de doenças infecciosas

- Fatores sociais e ambientais -

A seguir apresenta-se discussão do comportamento de variáveis relevantes na determinação do estado nutricional.

\subsection{Poder de Compra da Família}

Provavelmente é a variável isoladamente mais importante na determinação do estado nutricional. Conceitualmente, pode-se aumentar o poder de compra de alimentos de uma família através de:

- aumento de sua renda

- redução dos preços dos alimentos

- redução do preço de outros itens do orçamento do consumidor (que não alimentos), tais como habitação, escola, saúde, vestuário

o que leva à discussão em separado das inter-relações entre nutrição e renda e nutrição e preços.

\subsection{Renda}

Conforme nota Levinson ${ }^{15}$, a análise das inter-relações entre renda e estado nutricional através de dados em "cross section", a nível internacional, tem mostrado três tendências:

a. Lei de Engel - à medida que a renda aumenta, a percentagem de renda alocada à alimentação diminui, embora o dispêndio total com alimentos aumen- te. Ocorre exceção nos grupos de renda muito baixa que, ao receberem incremento de renda, aumentam as proporções relativas à alimentação. Isso se verifica devido ao fato de que esses grupos se encontram numa situação nutricional bastante precária, destinando qualquer aumento de renda para melhor alimentação.

$O$ aumento do dispêndio em termos absolutos pode resultar na aquisição de alimentos mais caros, mas, em geral, resulta também na ingestão de mais alimentos.

b. Conforme aumenta a renda, a proporção de calorias fornecidas por cereais e tubérculos decresce e é substituída por proulutos de origem vegetal, ou animal, mais custosos. Espera-se, em geral, impacto positivo desta tendência em termos nutricionais, com a ingestão de dieta mais variada.

c. Conforme aumenta a renda, existe tendência de mudança em direção a alimentos processados, que são mais caros. Em termos gerais, esta tendência pode ser um impacto negativo.

O impacto nutricional geral do aumento de renda é positivo pois os efeitos negativos são mais do que compensados pelos positivos. 
CAMPINO, A.C.C. Aspectos sócio-econômicos da desnutrição no Brasil. Rev. Saúde públ., S. Paulo, 20:83-101, 1986.

Levinson ${ }^{15}$ observa que, enquanto para a população como um todo os acréscimos de renda quase automaticamente se transformam em maiores quantidades de alimento, e de melhor qualidade, essa relação entre nutrição e renda não é tão clara para crianças entre 6 e 24 meses de idade, devido a problemas como infecção, digestibilidade e, em alguns países e regiões, crenças relativas à alimentação infantil.

\subsection{Preços}

Obviamente, uma forma de aumentar a renda real dos consumidores consiste em, dado o nível de renda, reduzir os preços dos bens por eles utilizados, entre os quais se destacam, por sua importância no orçamento familiar, os gêneros alimentícios. Reduções do preço permitem às famílias - especialmente as menos favorecidas, dado o seu nível de renda - adquirir maior quantidade de alimentos e, consequientemente, aumentar seu aporte calórico e de nutrientes.

Muita ênfase tem sido dada à disponibilidade de alimentos. Para nossos propósitos ela está incorporada no conceito de preço.

\subsection{Extensão do Sistema de Atendimento de Saúde}

Mesmo que o indivíduo tenha ingerido a quantidade suficiente de alimentos, 0 impacto destes sobre o estado nutricional pode ser reduzido por problemas de saúde. Assim, é sabido que a criança com infecção gastrointestinal pode ter seu estado nutricional prejudicado pelas consequiências de tal infecção, quer sejam estas representadas por falta de apetite, perda sanguínea ou redução na absorção de nutrientes. Ademais, doenças infantis de natureza comum como é o caso do sarampo, podem, quando associadas à carência nutricional, levar até à morte.

\subsection{Fatores Sociais e Ambientais}

Dentre estes fatores pode-se destacar os relativos às condições sociais da família, traduzidos pelo nível de educação dos pais, origem destes (é de especial importância no caso de crianças, a origem da mãe) e os relativos às condições de moradia da família, entre as quais, avulta, nos meios urbanos de países em desenvolvimento ou subdesenvolvidos, o suprimento de serviços básicos de saneamento, como água e esgoto. $O$ fator saneamento, em vários estudos, tem-se destacado como importante determinante do estado nutricional, pois precárias condições de saneamento levam ao desenvolvimento de moléstias como as infecções intestinais, cujos efeitos nocivos sobre 0 estado nutricional são conhecidos.

\subsection{Crenças Maternas sobre Nutrição e Saúde}

Muitas práticas adotadas pela mãe com relação à nutrição de seus filhos são incorretas e podem comprometer o estado nutricional dessas crianças.

Aspecto importante relativo às práticas maternas de alimentação refere-se à adoção de alimentos sólidos em substituição ao leite materno. Em muitos casos, o aleitamento prolonga-se além dos seis meses, sem adequada suplementação. Em outros casos, a suplementação existe, mas é insuficiente em muitos nutrientes essenciais; muitas vezes, a retenção de alimentos mais ricos reflete associação subjacente, feita pelas mães, entre estes alimentos e a ocorrência de infecções diarréicas.

Tais práticas são atribuídas à tradição. Entretanto, deve-se notar que muitos problemas decorrem do abandono da tradição e do contato com pessoas de regiões mais desenvolvidas e industrializadas, levando, por exemplo, ao abandono do aleitamento materno. 
CAMPINO, A.C.C. Aspectos sócio-econômicos da desnutrição no Brasil. Rev. Saúde públ., S. Paulo, 20:83-101, 1986.

Um exemplo que pode ser citado, refere-se à situação em que a mãe substitui o aleitamento natural - devido à imitação de padrões culturais de outros povos ou de pessoas de classe social mais elevada — por administração de leite em pó; este, geralmente, é caro para os níveis de renda mais baixos, o que leva ao suprimento em doses inadequadas e, o que é mais grave, em virtude de precárias condições de saneamento, freqüentemente este leite é preparado com água contaminada, comprometendo, dessa forma, não só o estado nutricional, como o estado geral de saúde da criança.

\subsection{Conteúdo Nutritivo dos Alimentos}

Em geral, o alimento em si implica certo valor nutricional, embora diferenças entre grupos de produtos ou mesmo entre variedades de um mesmo grupo possam ser significantes.

\subsection{Doenças Infecciosas}

Mesmo que a criança receba alimentação adequada, sua eficácia em termos nutricionais pode ser comprometida devido à presença de infecção gastrointestinal.

A infecção pode prejudicar o estado nutricional, ou mesmo precipitar a desnutrição, de várias formas:

- pode reduzir a ingestão de alimentos através da perda de apetite;

- resulta em perda de nitrogênio, requerendo, portanto, aumento na ingestão de proteínas;

- quando a infecção decorre de parasitas intestinais, ela frequientemente conduz à perda de sangue levando à anemia;

- se a infecção causa diarréia, a absorção de nutrientes no intestino será reduzida;

- se os níveis de ingestão de proteína já forem baixos, a doença diarréica pode precipitar a ocorrência de Kwashiorkor.
Ademais, doenças infantis como o sarampo podem ter efeito drástico, quer precipitando uma desnutrição severa, quer tendo impacto mais grave sobre crianças cronicamente desnutridas.

Finalmente, a criança pode ser afetada pelo estado nutricional da mãe durante a gravidez e, em menor grau, durante a lactância.

\subsection{Programas de Alimentação Fora do Lar}

A alimentação recebida fora de casa, frequientemente via programas governamentais, pode ter influência (espera-se que positivas) sobre o estado nutricional das crianças.

\section{3 - FATORES ASSOCIADOS À SITUAÇĀO NUTRICIONAL E DE CONSUMO DE ALIMENTOS NO BRASIL}

A partir desse modelo de análise, serão expostas, a seguir, algumas das informações disponíveis para o caso brasileiro. A literatura brasileira existente sobre o assunto é mais ampla do que a coberta neste trabalho, devendo-se destacar em especial os estudos do Departamento de Nutrição da Faculdade de Saúde Pública da USP. Entretanto, como se procurou nesta exposição dar ênfase aos aspectos relativos à renda, preços e condições de saneamento, as fontes básicas foram $o$ estudo do grupo IMPEP/IPE, ${ }^{7}$ as análises sobre o ENDEF - Estudo Nacional de Despesa Familiar ${ }^{10}$ e trabalhos do autor desse artigo ${ }^{2,3}$.

\subsection{Importância do Nível de Renda na Determinação do Estado Nutricional}

O estudo realizado pelo grupo IMPEP/ IPE $^{7}$ (1975) sugere as seguintes características de ocorrência de desnutrição:

a. A ocorrência de desnutrição protéico-calórica (DPC) está associada de maneira mais forte à deficiência de natu- 
reza quantitativa do que à qualitativa. Esta característica decorre da observação de que a dieta fornecida às crianças, pelas mães de classe de renda mais baixa, era adequadamente balanceada mas a quantidade suprida de alimentos era inadequada.

b. A deficiência quantitativa estava basicamente associada à deficiência de renda, pois cerca de $60 \%$ da renda das famílias de menor nível sócio-econômico era gasta em alimentação.
Essas duas características sugerem ser o problema de DPC muito mais sensível à condição nível de renda da família (que influi na quantidade de alimentos disponíveis) do que ao seu nível de escolaridade (que influi na qualidade de dieta).

A DPC apresentava-se inversamente correlacionada com o nível de renda da família, quantitativa e qualitativamente (Tabela 1).

TABELA 1

Distribuição de crianças de 6 a 60 meses classificadas de acordo com seu estado nutricional por classe de renda (Município de São Paulo)

\begin{tabular}{lrrrrrr}
\hline \multicolumn{2}{c}{ Renda SMPC(**) } & & & & & \\
Estado & $0-0,5$ & $0,5-1,0$ & $1,0-1,5$ & $1,5-2,5$ & 2,5 ou + & Total \\
Nutricional(*) & 8 & 13 & 13 & 22 & 27 & 83 \\
\hline Sobre-Peso & 46 & 54 & 66 & 63 & 62 & 291 \\
Eutrófico & 37 & 32 & 20 & 15 & 11 & 115 \\
Desnutrido I & 8 & 1 & 1 & - & - & 10 \\
Desnutrido II & 1 & - & - & - & - & 1 \\
Desnutrido III & 100 & 100 & 100 & 100 & 100 & 500 \\
\hline Total & 100
\end{tabular}

(*) Estado nutricional classificado pelo critério de Gomez, segundo padrão de Harvard.

(**) Classe de renda expressa em Salários Mínimos Familiares per capita (SMPC).

Fonte: Sigulem 18 (1980).

Obs.: Foram coletadas informações relativas a 100 crianças em cada classe de renda, de forma que os números absolutos lidos verticalmente fornecem a percentagem dentro da classe de renda.

$\mathrm{Na}$ primeira classe de renda (0-0,5 SMPC - Salário mínimo per capita), aproximadamente uma entre cada duas crianças era atingida por DPC, caindo essa proporção para uma dentre cada três na segunda classe de renda e uma dentre cinco na terceira classe de renda. Considerando-se que a desnutrição do grau I corresponde à sua forma atenuada, poder-se-ia sugerir que a partir de 1,5 SMPC a gravidade do fenômeno do DPC desaparece (só existe DPC de grau I). Ademais, a decomposição do qui-quadrado relizada por Sigulem ${ }^{18}$ (1980), demonstrou que a proporção de desnutridas era significativamente maior entre as crianças cujas famílias tinham renda familiar per capita inferior a 0,5 e entre 0,5 e 1,0 SMPC. Para se ter uma idéia de representatividade do fenômeno, deve-se considerar que $54 \%$ da população paulistana auferia menos do que 1,5 salário mínimo de renda familiar per capita.

Outros estudos realizados no Brasil também realçam a importância do nível de renda. Assim, em trabalho que a Fundação IBGE realizou para o UNICEF, 
CAMPINO, A.C.C. Aspectos sócio-econômicos da desnutrição no Brasil. Rev. Saúde públ., S. Paulo, 20:83-101, 1986.

em $1982^{11}$ verificou-se que a proporção de crianças de um a 5 anos que apresentavam déficit ponderal e/ou estrutural decrescia consideravelmente à medida que aumentava a classe de despesa da família. A relação déficit ponderal e/ou estrutural para despesa fica bastante clara no caso do nordeste urbano, onde passa de quase $50 \%$ para $6 \%$, mas também se faz presente, embora as diferenças não sejam tão acentuadas nas demais regiões consideradas (Nordeste rural, São Paulo urbano, São Paulo rural) (Tabelas 2, 3).

\section{TABELA 2}

Proporção (\%) de crianças de 1 a 5 anos com déficit ponderal e/ou estrutural, segundo o estrato e a classe de despesa corrente anual per capita.

\begin{tabular}{lrrrr}
\hline & \multicolumn{4}{c}{ Classe de despesa } \\
\cline { 2 - 5 } \multicolumn{1}{c}{ Estratos } & \multicolumn{1}{c}{$\mathbf{2}$} & \multicolumn{1}{c}{3} & 4 \\
\hline Nordeste urbano & 49,1 & 31,5 & $\mathbf{1 7 , 2}$ & 6,4 \\
Nordeste rural & 50,8 & 40,8 & 36,8 & 26,6 \\
São Paulo urbano & $\mathbf{1 8 , 2}$ & $\mathbf{8 , 1}$ & $\mathbf{6 , 2}$ & 4,4 \\
São Paulo rural & $\mathbf{2 2 , 8}$ & 16,1 & 10,0 & 12,0 \\
\hline
\end{tabular}

Fonte: FIBGE/UNICEF11 (1982).

TABELA 3

Classes de despesas corrente familiar per capita anual consideradas no ENDEF realizado no período 1974-1975.

\begin{tabular}{lcccc}
\hline & \multicolumn{5}{c}{ Classe de despesa (Cr\$) } \\
\cline { 2 - 5 } \multicolumn{1}{c}{ Estratos } & 1 & \multicolumn{1}{c}{2} & \multicolumn{1}{c}{3} & \multicolumn{1}{c}{ ( } \\
\cline { 2 - 5 } & até 1.380 & $1.381-2.274$ & $2.275-4.175$ & $4.176 \mathrm{e}+$ \\
Nordeste urbano & até 822 & $833-1.220$ & $1.221-1.818$ & $1.819 \mathrm{e}+$ \\
Nordeste rural & até 3.652 & $3.653-6.085$ & $6.086-10.826$ & $10.827 \mathrm{e}+$ \\
São Paulo urbano & até 1.810 & $1.811-2.561$ & $2.562-3.855$ & $3.856 \mathrm{e}+$ \\
São Paulo rural &
\end{tabular}

Obs.: As classes de despesa correspondem a, aproximadamente:

Até 0,5 salário mínimo per capita de 0,5 a 1,0 salário mínimo per capita mais de 1,0 salário mínimo per capita

Em termos de adequação calórica e protéico-calórica, estudo posterior de $\mathrm{Si}$ gulem ${ }^{19}$ (1981), com 191 famílias e igual número de crianças com idade inferior a 10 meses (crianças que foram objeto de seguimento durante 9 meses), mostrou que as famílias com nível de renda inferior a 0,5 SMPC apresentavam elevada proporção de casos com inadequação calórica e protéico-calórica $(82 \%)$ e que este nível era bastante superior ao verificado nas duas outras classes de renda pesquisadas, embora estas também configurassem uma população pobre (Tabela 4).

TABELA 4

Distribuição das famílias quanto à inadequação calórica e protéico-calórica da dieta segundo a classe de renda

\begin{tabular}{|c|c|c|c|c|c|c|c|c|}
\hline \multirow{2}{*}{$\begin{array}{c}\text { Classe de } \\
\text { Renda } \\
\text { (SMPC) }\end{array}$} & \multirow[b]{2}{*}{ n. ${ }^{\circ}$} & \multirow{2}{*}{$\begin{array}{l}\begin{array}{l}\text { N. }{ }^{\circ} \text { de } \\
\text { famílias }\end{array} \\
\%\end{array}$} & \multicolumn{2}{|c|}{ calórica } & \multicolumn{2}{|c|}{$\begin{array}{l}\text { Inadequação } \\
\text { protéico-calórica }\end{array}$} & \multicolumn{2}{|c|}{ Total } \\
\hline & & & n..$^{\circ}$ & $\%$ & n. ${ }^{\circ}$ & $\%$ & n. ${ }^{\circ}$ & $\%$ \\
\hline \begin{tabular}{r|l}
0 & $-0,5$ \\
0,5 & $-1,0$ \\
1,0 & $-1,25$
\end{tabular} & $\begin{array}{r}90 \\
84 \\
17 \\
191\end{array}$ & $\begin{array}{l}100 \\
100 \\
100 \\
100\end{array}$ & $\begin{array}{r}51 \\
37 \\
8 \\
96\end{array}$ & $\begin{array}{l}56,6 \\
44,1 \\
47,0 \\
50,3\end{array}$ & $\begin{array}{r}23 \\
8 \\
2 \\
33\end{array}$ & $\begin{array}{r}25,6 \\
9,5 \\
11,8 \\
17,3\end{array}$ & $\begin{array}{r}74 \\
45 \\
10 \\
129\end{array}$ & $\begin{array}{l}82,2 \\
53,6 \\
58,8 \\
67,6\end{array}$ \\
\hline
\end{tabular}

Fonte: Sigulem ${ }^{19}$ (1982). 
CAMPINO, A.C.C. Aspectos sócio-econômicos da desnutrição no Brasil. Rev. Saúde públ., S. Paulo, 20:83-101, 1986.

No Brasil, os dados do ENDEF ${ }^{10}$ mostram o consumo calórico e protéico por comensal-dia, que vem a ser um consumo per capita ajustado por um índice de presença às refeições. Verifica-se pela Tabela 5 nítida tendência do crescimento, quer do consumo calórico, quer do protéico, à medida que aumenta a classe de despesa em todas as regiões consideradas*.

Este quadro apresenta outros elementos adicionais que merecem ser discutidos. Tomando-se a necessidade média de calorias e proteínas calculada para o Brasil por Martins ${ }^{16}$ (1979) como sendo de 2.400 calorias/dia e 52,6 gramas de proteína, verificamos que:

a. as pessoas de classe de despesa mais baixa apresentam consumo calórico e protéico inferior à necessidade em todas as regiões;

b. a partir da classe de despesa per capita anual de Cr\$2.260 existe adequação protéica em todas as regiões, porém;

c. a adequação calórica se verifica para a classe de despesa 2 nas Regiões III (Paraná, Santa Catarina e Rio Grande do Sul) e IV (Minas Gerais e Espírito Santo) e para a classe de despesa 3 nestas duas regiões e mais as I (Rio de Janeiro) e II (São Paulo). Portanto, apresentavam inadequação calórica em todas as classes de renda as regiões $\mathrm{V}$ (Nordeste), VI (Distrito Federal) e VII (Norte-Centro-Oeste).

Estes dados configuram uma outra dimensão da relação alimentação-renda, que é a dimensão regional, em que as diferenças de renda regional estão associadas a diferenças na capacidade de aquisição e ingestão de alimentos pelas respectivas populações. Confirmam o comentário que fizemos em outro trabalho, ao analisar as pesquisas de orçamento familiar realizadas pela Fundação Getúlio Vargas (FGV), ${ }^{8}$ no início da década de 1960 , de que a subnutrição no Brasil é de natureza mais calórica do que protéica, mais urbana do que rural, crescendo com o tamanho das cidades (uma dimensão não revelada acima) e mais aguda no Nordeste (conforme os dados da FGV), ${ }^{8}$ Norte e Centro-Oeste (conforme dados do ENDEF citados por Campino, ${ }^{1}$ 1977).

\subsubsection{O Comportamento Intra-Classe de Baixa Renda: a Hipótese do Limiar}

Fato que nos tem chamado a atenção é o de que para níveis de baixa renda não se tem verificado, algumas vezes, a relação crescente esperada entre nível de renda e estado nutricional ou adequação calórica e/ou protéica.

Um exemplo disto é o que se encontra no trabalho de Sigulem ${ }^{19}$ (1981), que ao analisar a distribuição de estado nutricional (definido pelo critério de Gomez) segundo a classe de renda, não encontrou diferença estatisticamente significante na frequiência de desnutrição protéico-calórica entre as classes de renda. Trabalho que estamos realizando com um grupo de 61 crianças cujos pais trabalham em indústrias na área do Município de São Paulo não fornece indicação da associação clara entre renda e estado nutricional segundo Gomez**. Ambos os estudos foram feitos para populações com nível de renda per capita inferior a 1,255 SM (Campino e col.5, 1982). Isto nos leva a apresentar a "hi-

- Os dados do ENDEF10 são apresentados por classe de despesa, não por classe de renda. Como a diferença entre os dois conceitos é a poupança e nas classes de baixa renda esta é zero ou muito próxima, as pessoas incluídas na classe de despesa mais baixa são as mesmas que seriam abrangidas se fosse utilizada a classe de renda mais baixa.

* Estas 61 crianças foram examinadas pelo Instituto de Medicina Preventiva da Escola Paulista de Medicina - São Paulo, SP. 


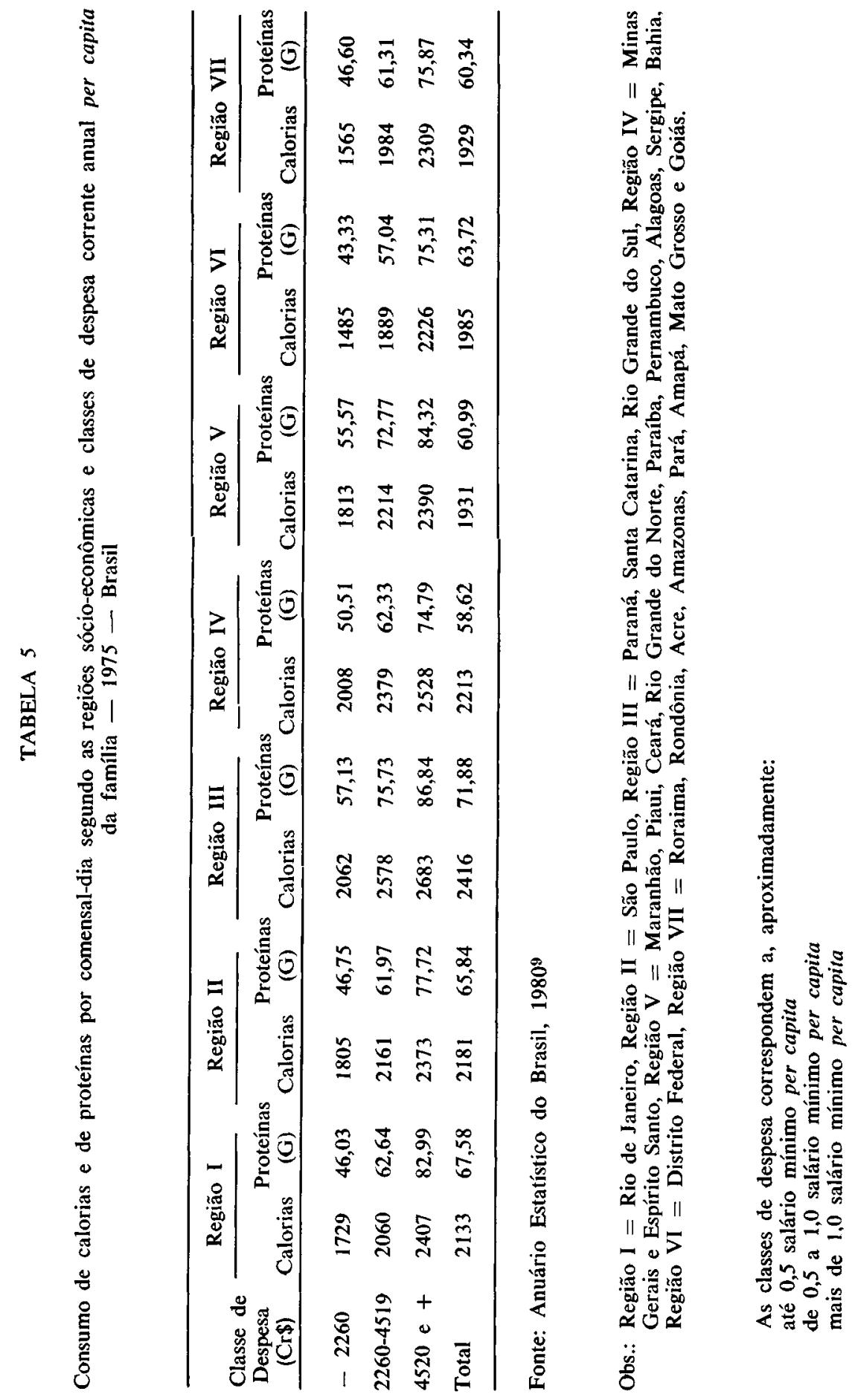


pótese do limiar" aplicada à relação estado nutricional-renda. De maneira muito simples esta hipótese significa que o estado nutricional de uma população (quer seja avaliado pela ingestão calórica e protéica da família, quer seja avaliada em termos de indicadores antropométricos para pré-escolares) não apresenta aumento estatisticamente significante até um certo nível de renda. A exposição precedente nos leva a situar este mínimo em 1,5 SMPC de forma que teríamos, simplificadamente, a relação observada na Figura.

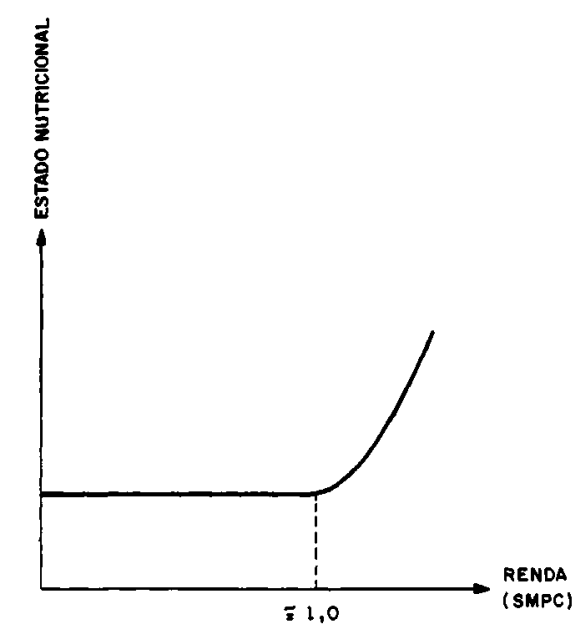

Figura - $\begin{gathered}\text { Relação entre estado nutricional e } \\ \text { renda. }\end{gathered}$

\subsection{Preços}

A bibliografia relativa ao impacto nutricional de variações nos preços de alimentos é bastante escassa entre nós. $O$ que se tem feito está ligado à questão da eficácia de políticas de subsídios aos preços de alimentos básicos, em termos nutricionais.
As informações disponíveis são no sentido de que uma redução no preço de alguns alimentos básicos - como arroz e feijão - teria muito provavelmente um impacto nutricional pouco significativo, embora possa ter um impacto redistributivo. A base para esta assertiva, escudada em estudo realizado para o INAN (Instituto Nacional de Alimentação e Nutrição) é a falta de elasticidade de oferta dos alimentos considerados, isto é, o fato de que a oferta destes alimentos varia menos que proporcionalmente às variações nos preços (Campino ${ }^{5}, 1982$, FIPE/INAN ${ }^{12}$, 1982). A seguir apresenta-se, em detalhes, a simulação realizada para o caso do arroz que fornece indicações de política semelhantes às obtidas para o feijão.

\subsubsection{Efetios Prováveis de Subsídio aos Preços do Arroz}

Utilizando-se de um conjunto de elasticidade-preço estimado por diversos autores, procurou-se verificar o impacto que níveis alternativos de subsídio teriam sobre os preços e quantidades de equilíbrio, se fossem estendidos a nível nacional. Como os autores estimaram suas elasticidades a partir de funções aritméticas e logarítmicas, utilizou-se apenas o valor obtido na especificação logarítmica.

As estimativas de elasticidades utilizadas para a demanda de arroz no Brasil são as de Sobral ${ }^{20}$ (1973) e Contador ${ }^{6}$ (1977) e para a oferta as de Pastore ${ }^{17}$ (1968), Contador ${ }^{6}$ (1977) e Gontijo ${ }^{13}$ $(1975)^{*}$.

A Tabela 6 apresenta os resultados obtidos da aplicação das elasticidades estimadas por estes autores. Em relação às

- O trabalho de Sobral20 é relativo ao Brasil e foi feito para o período $1950 / 1970.0$ estudo de Pastore17 foi feito para o Estado de São Paulo, ao passo que Gontijo ${ }^{13}$, embora trabalhando basicamente com o Estado de São Paulo, também apresenta resultados para o Nordeste. As estimativas de Contador6, que ele próprio designa como imaginosas, são relativas ao Brasil. 
CAMPINO, A.C.C. Aspectos sócio-econômicos da desnutrição no Brasil. Rev. Saúde públ., S. Paulo, 20:83-101, 1986.

quantidades, verifica-se que o maior impacto ocorreria no caso do subsídio de $60 \%$, se a elasticidade-preço da demanda fosse aquela estimada por Contador $^{6}$ $(=-0,20)$ e a elasticidade de oferta de longo prazo fosse maior do que a unidade $(1,39)$. Nesse caso, o aumento na quantidade de equilíbrio seria de $17 \%$ reduzindo-se para $14 \%$ no curto prazo (elasticidade de oferta $=0,55$ ). Entretanto, deve ser ressaltado que este resultado é bastante influenciado pela estimativa de elasticidade de demanda apresentada por Contador ${ }^{6}$, que parece elevada. Quando se trabalhou com os resultados de Sobral ${ }^{20}$, os impactos sobre a quantidade situavam-se sempre abaixo de $2 \%$.

TABELA 6

Efeitos de níveis diversificados de subsídio sobre preços e quantidades de equilíbrio — arroz

\begin{tabular}{|c|c|c|c|c|c|c|c|}
\hline \multicolumn{2}{|c|}{ Elasticidades } & \multicolumn{3}{|c|}{$\begin{array}{c}\text { Efeitos sobre o preço (P'/P) } \\
\text { Subsídios }\end{array}$} & \multicolumn{3}{|c|}{$\begin{array}{c}\text { Efeitos sobre a quantidade }\left(Q^{\prime} / Q\right) \\
\text { Subsídios }\end{array}$} \\
\hline Demanda & Oferta & $30 \%$ & $45 \%$ & $60 \%$ & $30 \%$ & $45 \%$ & $60 \%$ \\
\hline \multirow[t]{2}{*}{$-0,018^{4}$} & ${ }^{1} \mathrm{CP}=0,232$ & 1,0260 & 1,0440 & 1,068 & 1,0060 & 1,0100 & 1,0154 \\
\hline & $\mathrm{LP}=0,547$ & 1,0114 & 1,0190 & 1,030 & 1,0062 & 1,0105 & 1,0161 \\
\hline \multirow[t]{2}{*}{$-0,20^{5}$} & $C P=0,232$ & 1,1795 & 1,3189 & 1,5284 & 1,0228 & 1,0386 & 1,0598 \\
\hline & $\mathrm{LP}=0,547$ & 1,1002 & 1,1736 & 1,2780 & 1,0536 & 1,0915 & 1,1436 \\
\hline \multirow{6}{*}{$\begin{array}{l}-0,018^{4} \\
-0,20^{5} \\
-0,018\end{array}$} & $2 \quad 0,30$ & 1,0204 & 1,0344 & 1,0532 & 1,0061 & 1,0102 & 1,0157 \\
\hline & $\mathrm{SP}^{0,30}$ & 1,1533 & 1,2701 & 1,4421 & 1,0437 & 1,0744 & 1,1162 \\
\hline & $3 \mathrm{CP}=0,55$ & 1,0113 & 1,0191 & 1,0295 & 1,0062 & 1,0105 & 1,0161 \\
\hline & $\begin{array}{c}\mathrm{LP}=1,39 \\
\mathrm{NE}\end{array}$ & 1,0046 & 1,0077 & 1,0118 & 1,0063 & 1,0107 & 1,0164 \\
\hline & $C P=0,03$ & 1,1431 & 1,2513 & 1,4100 & 1,0040 & 1,0067 & 1,0104 \\
\hline & $\mathrm{LP}=0,10$ & 1,0559 & 1,0954 & 1,1500 & 1,0055 & 1,0092 & 1,0141 \\
\hline \multirow[t]{5}{*}{$-0,20^{5}$} & SP & & & & & & \\
\hline & $C P=0,55$ & 1,0998 & 1,1728 & 1,2768 & 1,0537 & 1,0916 & 1,1438 \\
\hline & $\begin{array}{c}\mathrm{LP}=1,39 \\
\mathrm{NE}\end{array}$ & 1,0459 & 1,0781 & 1,1222 & 1,0643 & 1,1102 & 1,1737 \\
\hline & $C P=0,03$ & 1,3636 & 1,6818 & 2,2184 & 1,0093 & 1,0157 & 1,0242 \\
\hline & $\mathrm{LP}=0,10$ & 1,2684 & 1,4897 & 1,8420 & 1,0241 & 1,0407 & 1,0630 \\
\hline
\end{tabular}

Fonte: FIPE12 (1982).

Obs.: Demanda: Estimativas de Sobral20 (1973) e Contador ${ }^{6}$ (1977).

Oferta: Estimativas de Pastore17 (1968), Contador ${ }^{6}$ (1977) e Gontijo13 (1975).

Considerando-se que Sobral ${ }^{20}$ derivou suas estimativas de trabalho de natureza econométrica e que Contador ${ }^{6}$ derivou suas estimativas de discussão com técnicos agrícolas, denominando-as de "imaginosas", conclui-se que a aplicação de uma política do tipo PINS (Projeto Integrado de Nutrição e Saúde) a nível nacional teria muito provavelmente impactos modestos sobre as quantidades de equilíbrio, sendo mais confiável a utilização das estimativas de Sobral. ${ }^{20} \mathrm{O}$ impacto sobre a quantidade de equilíbrio somente se aproximaria de $10 \%$ se a elasticidade de demanda fosse $-0,2$ e as elasticidades de oferta fossem semelhantes àquelas estimadas por Gontijo ${ }^{13}$ para São Paulo, com níveis de subsídio iguais ou superiores a $45 \%$ e atingindo no máximo $17 \%$ quando o nível de subsídio fosse de $60 \%$.

Note-se que no caso do Nordeste as informaçōes disponíveis permitem prever impacto bastante modesto sobre a quantidade ofertada, qualquer que seja a elasticidade de demanda considerada, 
CAMPINO, A.C.C. Aspectos sócio-econômicos da desnutrição no Brasil. Rev. Saúde públ., S. Paulo, 20:83-101, 1986.

atingindo no máximo $6 \%$, caso o subsídio fosse de $60 \%$. Isto se deve à estimativa de oferta bastante inelástica para a região.

Analisando-se o comportamento dos prelos, verifica-se que os aumentos previstos são inferiores ao nível de subsídio, em todas as situações, exceto para o Nordeste, quando a elasticidade de oferta estimada para a região é combinada com a elasticidade de demanda de $-0,2$, situação em que o efeito sobre os preços é próximo, ou superior, ao nível de subsídio.

A análise precedente leva a concluir que um programa de subsídio de preço, se executado a nível nacional, provavelmente teria efeitos redistributivos importantes, na medida em que provoca aumentos de preços na mailoria das vezes inferiores ao nível de subsídio e portanto permite transferência de renda real para a população beneficiada, mas teria efeitos pouco significativos sobre a quantidade ofertada, portanto, teria impacto nutricional nulo. Exceções a este provável comportamento geral seriam encontradas caso a oferta a nível de Brasil apresentasse um grau de inelasticidade semelhante ao da oferta no Nordeste, situação em que os efeitos redistributivos sugeridos não se verificariam, ou caso a oferta apresentasse uma reação aos preços semelhante à da agricultura paulista, no qual a quantidade reagiria de maneira significativa; ambas as hipóteses parecem pouco prováveis.

Uma possível limitação ao procedimento utilizado decorre da hipótese explícita de que um programa de subsídio de preços conduzido ao nível nacional beneficiaria indiscriminadamente toda a população. Pode-se imaginar, em princípio, que o programa fosse eficaz em beneficiar apenas a população de baixa renda e que esta exclusivamente se beneficiasse dos aumentos de quantidade verificados, de forma que o programa teria um impacto nutricional positivo sobre a população de baixa renda. Esta possibilidade depende exclusivamente da condição de implantarse a nível nacional um programa de subsídio de caráter discriminativo em relação à população com nível de renda superior a dois salários mínimos; entretanto, esta possibilidade é difícil de se concretizar a nível prático em um programa nacional.

Outra limitação decorre do fato de que as elasticidades de demanda utilizadas são válidas para a população como um todo, havendo a possibilidade de que para a população de baixa renda as elasticidades-preço de demanda fossem substancialmente superiores às verificadas para a média da população, gerando efeitos nutricionais positivos. Entretanto, o teste realizado pelo autor, para o caso do feijão, com dados relativos à população de baixa renda: de Recife, não confirma esta hipótese (FIPE/INAN ${ }^{12}$, 1982).

\subsection{Condições Familiares - Condições de Emprego}

Dada a relação conhecida entre renda e estado nutricional da criança em idade pré-escolar, um̧a informação útil do ponto de vista de orientação de programas de massa, que objetivem minorar a situação carencial dessas crianças, seria a distribuição dos chefes de família por setor de atividade e/ou nível de ocupação.

Utilizando os dados colhidos no estudo IMPEP/IPE ${ }^{7}$ (1975), verifica-se que grande parte dos chefes de famílias, cujos niveis de renda eram iguais ou inferiores a 1,0 SMPC, desempenhavam atividades no setor terciário, em ocupação de baixo nível de produtividade. Por outro lado, há uma grande percentagem de indivíduos nas classes de renda mais altas também nesse setor, mas estes desempenham atividades que requerem maior qualificação e, portanto, têm um nível ocupacional de 
CAMPINO, A.C.C. Aspectos sócio-econômicos da desnutrição no Brasil. Rev. Saúde públ., S. Paulo, 20:83-101, 1986.

maior prestígio. Já em relação ao setor secundário, são as classes 2 e $3(0,5-$ 1,5 SMPC) as que contam com maior percentagem de indivíduos ocupados, o que, possivelmente, indicava a necessidade de mão-de-obra mais qualificada pelas indústrias do ramo moderno.

No que se refere à ocupação, os dados foram codificados de acordo com a escala de prestígio elaborada por HutchinsonGouveia ${ }^{14}$, onde os códigos estão inversamente relacionados com o prestígio ocupacional.

Verifica-se pela Tabela 7 que há um estreito relacionamento entre $o$ nível ocupacional e o nível de renda familiar. $O$ quadro apresenta uma disposição em diagonal com maior participação de indivíduos que exercem ocupações manuais e de pouco prestígio nas primeiras classes de renda; conforme a renda se eleva, os chefes de família ocupam posições de maior prestígio.

Os dados foram agrupados no quadro de modo que o primeiro estrato (ocupações 1 e 2) represente ocupações de elevado status social; no estrato seguinte encontram-se atividades de supervisão e ocupações não- manuais; o estrato inferior engloba as ocupações manuais. Verifica-se que para as famílias, cuja renda era inferior a 1,5 SMPC a maior incidência de desnutrição ocorrida foi justamente naquelas cujos chefes exerciam ocupações manuais. A partir de 1,5 SMPC, a maior proporção de crianças desnutridas aparece nas famílias nas quais o pai exercia ocupações não-manuais de rotina ou posições mais baixas de supervisão.

A mesma constatação feita sobre a ocupação do pai é válida para as mães: nas classes de renda mais baixas, exercem ocupações de menor prestígio - na maioria como empregadas domésticas enquanto nas classes de renda mais alta dedicam-se a ocupações qualificadas.

Para o impacto nutricional devido ao trabalho fora do lar, das mães de menor nível de renda, duas hipóteses têm sido formuladas:

a) A mãe que trabalha fora dedica menos tempo às atividades do lar e, em conseqüuência, a criança em idade préescolar terá maior probabilidade de ser mal alimentada.

b) O fato de as mães de famílias de nível mais baixo de renda exercerem ocupações de empregada doméstica e fazerem refeições no local de emprego, permite uma realocação dos gastos com alimentação, contribuindo positivamente para o estado nutricional familiar e, inclusive, do pré-escolar.

$O$ efeito líquido será o resultante destes dois efeitos.

Entretanto, tanto no estudo do IM$\mathrm{PEP} / \mathrm{IPE}^{7}$ quanto no trabalho relativo à alimentação do trabalhador (Campino e col. $\left.{ }^{5}, 1982\right)$ não se verificou qualquer relação estatiscamente significante entre estado nutricional e o fato de a mãe trabalhar fora.

Antes de terminar esta breve discussão sobre a situação de emprego, deve-se enfatizar a importância do acesso a um emprego no setor formal como um determinante do estado nutricional. Assim, no estudo acima mencionado (Campino e col.5, 1982), em que todas as crianças examinadas pertenciam a famílias com nível de renda inferior a 1,2 SMPC, mas cujos chefes dispunham de um emprego formal no setor industrial, observou-se maior proporção de crianças normais do que no trabalho IMPEP/IPE ${ }^{7}$ (1975), e, o que é mais importante, nenhum caso de desnutrição de II e III grau. Embora oito anos tenham decorrido entre um estudo e outro, nossa sugestão é que em parte a diferença pode ser atribuída ao fato de que uma parcela dos chefes de família cujas crianças foram examinadas em 1974-75 não tinham empregos no setor formal e dedicavam-se a atividades 
CAMPINO, A.C.C. Aspectos sócio-econômicos da desnutrição no Brasil. Rev. Saúde públ., $\mathrm{S}$. Paulo, 20:83-101, 1986.

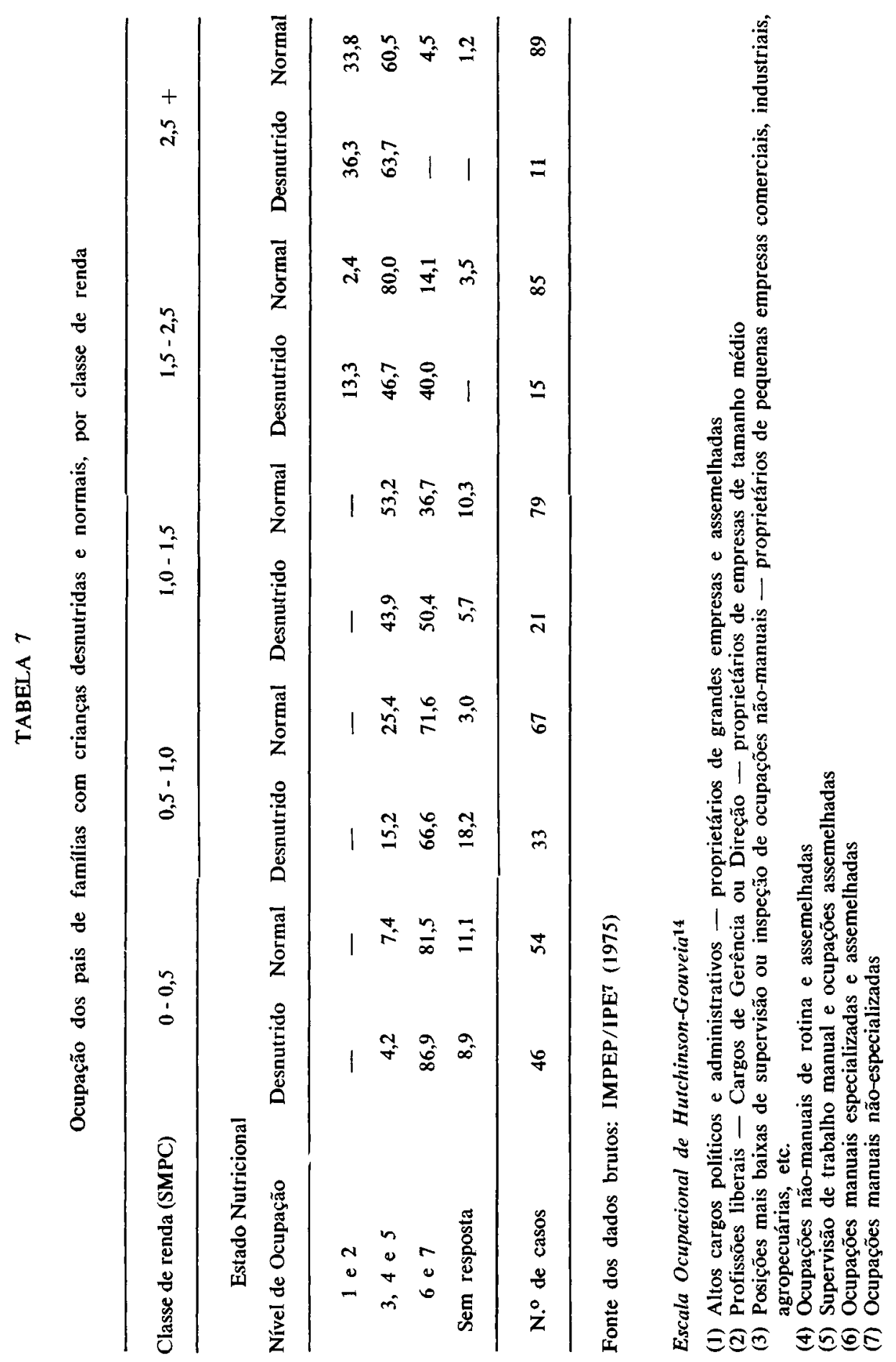


CAMPINO, A.C.C. Aspectos sócioeconômicos da desnutrição no Brasil. Rev. Saúde públ., S. Paulo, 20:83-101, 1986.

informais no terciário (biscateiro, por exemplo). A Tabela 8 ilustra este ponto.

\section{TABELA 8}

Comparação entre um estudo com crianças cujos pais estavam empregados no setor secundário formal e um grupo populacional mais amplo

\begin{tabular}{lcc}
\hline & $\begin{array}{c}\text { Crianças de famílias cujos chefes } \\
\text { estavam empregados }\end{array}$ \\
\cline { 2 - 3 } $\begin{array}{l}\text { Estado } \\
\text { nutricional }\end{array}$ & $\begin{array}{c}\text { Exclusivamente no } \\
\text { secundário } \\
\text { formal }\end{array}$ & $\begin{array}{c}\text { Secundário e } \\
\text { terciário } \\
\text { formal e } \\
\text { informal }\end{array}$ \\
\hline $\begin{array}{l}\text { Sobre-peso } \\
\text { Eutrófico }\end{array}$ & $3,3 \%$ & $16,6 \%$ \\
$\begin{array}{l}\text { Desnutrição } \\
\text { I Grau }\end{array}$ & $65,6 \%$ & $58,2 \%$ \\
$\begin{array}{c}\text { Desnutrição } \\
\text { II Grau }\end{array}$ & $31,1 \%$ & $23,0 \%$ \\
$\begin{array}{c}\text { Desnutrição } \\
\text { III Grau }\end{array}$ & - & $2,0 \%$ \\
\hline N. ${ }^{\circ}$ de Classes & 61 & $0,2 \%$ \\
\hline
\end{tabular}

Fonte: Campino e col.5 (1982), Sigulem ${ }^{19}$ (1981)

\subsection{Condições de Moradia: Saneamento}

Verificou-se no trabalho IMPEP/IPE ? que existe uma estreita correlação entre a prevalência de anemias e a desnutrição protéico-calórica bem como entre verminoses e desnutrição protéico-calórica.

Este fato indica que um dos fatores que deveria merecer ênfase em uma política que visasse à redução da prevalência de desnutrição protéico-calórica seria a condição de saneamento.

Verificou-se que (IMPEP/IPE ${ }^{7}$ ) 0 número de domicílios que recebem água através da rede pública varia proporcionalmente ao nível de renda. Apenas 36\% das famílias de classe de renda mais bajxa têm seus domicílios ligados à rede pública e $60 \%$ se utilizam de poços e/ou minas. Melhoria substancial verifica-se na classe seguinte, na qual $62 \%$ das famílias estão ligadas à rede de abastecimento de água.

Quanto a destinação dos dejetos, na média, $56,8 \%$ da população amostrada naquele estudo residia em domicílios ligados à rede de esgotos e $41,4 \%$ das famílias se utilizavam de fossa. Verificase, também, que as condições de destinação de dejetos variam diretamente com a renda. $\mathrm{Na}$ faixa de até $0,5 \mathrm{SMPC}$ apenas $32 \%$ das famílias têm sua residência ligada à rede de esgoto, chegando essa percentagem a $85 \%$ na classe de renda mais elevada.

TABELA 9

Condições de abastecimento de água e de destinação dos dejetos nos domicílios segundo o nivel de renda das familias

\begin{tabular}{|c|c|c|c|c|c|c|}
\hline \multirow{2}{*}{$\begin{array}{l}\text { Nível de renda } \\
\text { Condição de abastecimento } \\
\text { água e destinação de dejetos }\end{array}$} & \multicolumn{6}{|c|}{ Percentagem dos domicílios } \\
\hline & $0-0,5$ & $0,5-1,0$ & $1,0-1,5$ & $1,5-2,5$ & 2,5 ou + & Total \\
\hline $\begin{array}{l}\text { Domicílios ligados à rede pú- } \\
\text { blica }\end{array}$ & 38,0 & 62,0 & 73,0 & 90,0 & 92,0 & 71,0 \\
\hline $\begin{array}{l}\text { Domicílios não ligados à rede } \\
\text { pública (poço ou mina) }\end{array}$ & 60,0 & 36,0 & 27,0 & 10,0 & 7,0 & 28,0 \\
\hline Torneira ligada à rede pública & 2,0 & 2,0 & - & - & 1,0 & 1,0 \\
\hline $\begin{array}{l}\text { Domicílios ligados à rede de } \\
\text { esgoto } \\
\text { Não ligados: }\end{array}$ & 32,0 & 37,0 & 58,0 & 72,0 & 85,0 & 56,8 \\
\hline $\begin{array}{l}\text { Não ligados: } \\
\text { - com fossa } \\
\text { - outros }\end{array}$ & $\begin{array}{r}66,0 \\
2,0\end{array}$ & $\begin{array}{r}57,0 \\
6,0\end{array}$ & $\begin{array}{r}41,0 \\
1,0\end{array}$ & 28,0 & 15,0 & $\begin{array}{r}41,4 \\
1,8\end{array}$ \\
\hline
\end{tabular}

Fonte: IMPEP/IPE7 (1975). 
CAMPINO, A.C.C. Aspectos sócio-econômicos da desnutrição no Brasil. Rev. Saúde públ., S. Paulo, 20:83-101, 1986.

Os dados constantes da Tabela 9 evidenciam a situação precária das famílias de nível baixo de renda quanto às condições de saneamento básico. Pode-se supor que esta situação afeta o estado nutricional das crianças, pelo favorecimento de transmissão de doenças que podem dificultar o aproveitamento biológico dos alimentos. Não obstante a disponibilidade destes serviços tenha melhorado substancialmente de 1975 até o presente, ainda resta um significativo diferencial entre classes de renda.

\subsection{Condições Gerais de Saúde e Hábitos Alimentares}

Embora não estejam reproduzidas neste trabalho, encontram-se no estudo IMPEP/IPE 7 informações sobre o tipo de equipamento de saúde mais utilizado. Observa-se naquele estudo que a população de baixa renda utiliza-se proporcionalmente mais dos Centros de Saúde $(74 \%)$, vindo em segundo lugar o INPS (Instituto Nacional de Previdência Social) - $61 \%$. Assim, embora se possa discutir a conveniência de localização dos Centros de Saúde, o fato importante, do ponto de vista do planejamento de programas que visem reduzir a incidência de subnutrição, é que este tipo de equipamento de saúde é amplamente utilizado pela clientela básica de programas desta natureza*.

Quanto aos aspectos relativos a tradições e comportamentos da mãe, existe um resultado bastante interessante e ilustrativo no trabalho IMPEP/IPE ${ }^{7}$ referente à situação de aleitamento. Verificou-se que o período modal de aleitamento era inferior ou igual a um mês $(46,1 \%)$. Entretanto, quando inquiridas em outra parte do questionário sobre o período que achavam que deveria durar a amamen- tação, 90\% das mães respondiam 6 meses. Esta constatação ilustra bem o aspecto de que este não é um problema de educação, porque mestno as mulheres analfabetas têm bem presente a idéia de que a amamentação nos primeiros 6 meses de vida é necessária.

\section{4 - ALGUMAS REFLEXÕES SOBRE UMA POLITICA DE NUTRIÇÃO}

Nas páginas precedentes confirma-se que o principal determinante do estado nutricional é a renda per capita. Entretanto, quando se deseja propor uma política que vise melhorar o estado nutricional de nossa população, a primeira pergunta que aflora é: qual a magnitude do aumento de renda necessário para gerar uma correção dos déficits nutricionais?

Os dados constantes da Tabela 4 para a classe de renda de até 0,5 SMPC permitem verificar que, à exceção de Brasília, o aumento na ingestão calórica necessária era da ordem de $30 \%$ a $40 \%$. Como a elasticidade-renda do consumo de calorias é de aproximadamente 0,3 , isso implica aumentos de renda da ordem de $100 \%$ a $133 \%$. Ora, esta população despende entre 0,4 e 0,7 SMPC por ano. Quando se considera que cerca de $20 \%$ da população economicamente ativa, em 1976, recebia salário inferior a este nível, pode-se avaliar a implicação de conceder aumentos de salário real de $100 \%$ ou mais para um contingente desta ordem.

Com isto, não pretendemos dizer que a solução do problema nutricional não deva passar pela distribuição de renda. Cremos que os resultados precedentes deixaram claro que a melhor política de nutrição é uma política de distribuição de renda e de geração de empregos. En-

- O autor orientou um estudo no qual se procurou relacionar a localização dos equipamentos de saúde e a dos desnutridos, segundo o estudo IMPEP/IPE7. Entretanto, não foi possível verificar um padrão de localização na medida em que as crianças desnutridas estavam espalhadas em todos os bairros do Município de São Paulo. 
CAMPINO, A.C.C. Aspectos sócio-econômicos da desnutrição no Brasil. Rev. Saúde públ., S. Paulo, 20:83-101, 1986.

tretanto, a magnitude da renda adicional a ser distribuída às classes de baixa renda é de tal ordem que esta política requererá, necessariamente, um período longo para que seus efeitos sejam sentidos. Distribuição de renda é, do ponto de vista nutricional, uma política de longo prazo; existem alguns sinais de que está ocorrendo um processo de desconcentração de renda, mas seus impactos nutricionais verificar-se-ão apenas em um prazo mais longo, dada a magnitude da redistribuição necessária.

Em relação ao emprego, os dados apontam para as consequiencias negativas e para o elevado custo social de uma política recessiva. Se houver recessão econômica e consequiente desemprego, sem dúvida o estado nutricional da população piorará e este é um motivo suficiente para uma política recessiva não ser implantada. Como subsídios a preços de alimentos são pouco eficazes do ponto de vista nutricional, dada a inelasticidade da oferta, restam os programas de suplementação alimentar. Estes, embora conhecido seu caráter paliativo, são ainda importantes. A literatura internacional mostra que não se pode, com convicção, associar estes programas à melhorias significativas de estado nutricional das populações assistidas, mas pela dificuldade em especificar-se adequadamente grupos-controle, fica presente a possibilidade de a situação dessa população ser pior não fosse o programa; idêntica observação foi feita quando da análise do PINS, conduzido pelo Centro Integrado de Saúde Amauri de Medeiros da Fundação de Ensino Superior de Pernambuco (CISAM/FESP) em Pernambuco com recursos do INAN.

Entretanto, para estes programas serem eficazes é necessário, a nosso ver (Campino, ${ }^{2}$ 1979):
- aumentar os recursos disponíveis para a área de alimentação e nutrição. Em estimativa que fizemos, calculamos que a atenção adequada à população urbana de nível de renda inferior a um salário mínimo requeria, a nível federal, que os $1 \%$ dedicados à alimentação e nutrição fossem quintuplicados;

- melhorar a eficiência dos atuais programas. Alguns programas são muito caros e o seu barateamento requer que se enfrente a questão do grau de processamento julgado desejável;

- reduzir o número de programas, para realizar ganhos de escala, já que os recursos disponíveis estão pulverizados numa grande gama de programas.

Isto requer uma mudança de visão filosófica do problema em que o enfoque de grupos biologicamente vulneráveis que levou à um "fatiamento" da atenção dedicando-se diferentes segmentos do problema nutricional a diferentes Ministérios - para um enfoque de grupos socialmente vulneráveis em que a atenção seja dada à família de baixa renda.

Dentro deste enfoque é oportuno analisarmos o Programa de Prioridades Sociais, que está sendo desenvolvido pelo atual Governo. Este programa foi contemplado, em maio de 1985, com Cr\$ 3.838 trilhões (US\$ 733 milhões)*.

O programa mais importante desta área é o PNS - Programa de Nutrição e Saúde - para o qual estão previstos Cr\$ 1.552 trilhões (US\$ 296 milhões) e uma população-alvo de 11,6 milhões de pessoas. Isto implicaria dispêndio da ordem de Cr\$ 11.153 (US\$ 2,13) pessoa/mês, recursos que serão repassados aos beneficiários sob a forma de uma cesta básica da qual constam arroz, feijão, leite, açúcar, fubá e farinha de mandioca. O custo básico dessa cesta, que calculamos

\footnotetext{
* Os valores foram calculados na base de Cr\$5.236 o dólar, câmbio de maio de 1985 .
} 
CAMPINO, A.C.C. Aspectos sócio-econômicos da desnutrição no Brasil. Rev. Saúde públ., S. Paulo, 20:83-101, 1986.

com base nos dados do Indice de Custo de Vida (ICV/FIPE), é de Cr\$ 816,13 (US\$ 0,11)/1.000 calorias, de forma que permitiriam destinar por dia 455 calorias/ beneficiário, correspondendo, grosso modo, a $19 \%$ das necessidades diárias. Embora um programa de suplementação alimentar, para ser significativo, deva cobrir $30 \%$ das necessidades diárias, a especificação dada ao programa parece razoável, se comparada ao desempenho do PNS até o momento. Por outro lado, o conjunto de produtos escolhidos para compor a cesta básica é bastante significativo em termos dos hábitos alimentares da população brasileira.

O Programa de Abastecimento Popular (PROAB) ampliou essa cesta básica para 12 produtos, adicionando aos anteriormente definidos o óleo comestível, carnes, pescados, ovos, macarrão e café. Pretende cobrir 13,2 milhões de pessoas, o que parece ser uma meta exagerada quando se considera que o programa atualmente existente, atinge apenas $2 \mathrm{mi}$ lhões de pessoas. Por outro lado, os recursos alocados ao programa são pouco expressivos, $\mathrm{Cr} \$ 87,7$ bilhões (US\$ 16,749 milhões) de sorte que sua redistribuição à população-alvo corresponderia a Cr\$ 550/beneficiário/mês. Como a cesta ampliada para 12 produtos apresenta um custo de $\operatorname{Cr} \$ 17.881$ (US\$ 3,41)/ 1.000 calorias, esses recursos permitiram adquirir 28 calorias/mês. Este resultado decorre da inclusão do café no conjunto de produtos a serem subsidiados neste programa, quando este apresenta apenas 41 calorias por quilo. Excluindo-se o café, o custo por 1.000 calorias passa a $\operatorname{Cr} \$ 1.549$ (US\$ 0,29) e os recursos do programa permitiriam distribuir 355 calorias/beneficiário/mês ou $11,8 / \mathrm{dia}$, menos de $0,5 \%$ das necessidades diárias. E, portanto, um programa pouco expressivo em face da natureza do problema, não havendo, ademais, no seu enunciado, qualquer esclarecimento sobre a forma como se pretende operá-lo como um programa de subsídio de preço (por exemplo, qual o nível do subsídio e a quem é repassado).

O Reforço Alimentar de Creches é, como o nome diz, um reforço aos programas de creches-casulo da Legião Brasileira de Assistência (LBA), não havendo menção à introdução de quaisquer modificações decorrentes da experiência passada. Ademais, o recurso implícito por beneficiário, de Cr $\$ 6.301 /$ mês (US\$ 1.20 ), difere daquele mencionado no texto do programa (Cr\$15.000) (US\$2.86).

E lamentável que o Programa de Alimentação do Trabalhador não tenha tido sua especificação revista antes da edição deste Programa de Prioridades Sociais, sendo apresentado como um programa em reestudo. Este programa era, em 1983, o mais significativo dos programas de alimentação e nutrição, absorvendo $38 \%$ dos recursos do PRONAN (Programa Nacional de Alimentação e Nutrição).

Analisando o conjunto dos programas de alimentação e nutrição incluídos no Programa de Prioridades Sociais, estranhamos que a preocupação básica foi apenas a de propiciar um reforço de verbas para o setor, sem ter havido uma reflexão mais aprofundada sobre a reorientação desses programas. Esta reflexão deveria incorporar as informações já conhecidas sobre a eficiência e eficácia dos mesmos e procurar evitar os problemas já conhecidos nesta área, como a superposição de ações, a falta de capacidade gerencial para a condução dos programas, a inexistência de uma coordenação efetiva para os mesmos, a pulverização dos recursos e a ocorrência de deseconomias de escala, decorrente do grande número de programas. Não atentar para estas dificuldades reais pode, simplesmente, ter como decorrência a má utilização dos recursos destinados a esta área. 
CAMPINO, A.C.C. Aspectos sócio-econômicos da desnutrição no Brasil. Rev. Saúde públ., S. Paulo, 20:83-101, 1986.

\section{AGRADECIMENTO}

O Autor agradece as críticas e sugestões dos relatores da Revista de Saúde Pública, que procurou incorporar. Como sempre, as falhas e imperfeições remanescentes são de exclusiva responsabilidade do autor.

CAMPINO, A.C.C. [Socio-economic aspects of subnutrition in Brazill, Rev. Saúde públ., S. Paulo,20:83-101, 1986.

\begin{abstract}
It is proposed here to offer a general view of the social and economic factors that have been identified as the main determinants of a country's nutritional situation. The conclusion is reached that income, considered in isolation, is the most important factor in determining the nutritional condition, but once this variable is fixed, other factors such as the extent of the health attendance system, educational level, food and nutrition programs - also play an important part. An attempt is made impirically to decide, which are the determinants of the nutritional situation for Brazil, in the light of previous research. It becomes evident, as the literature leads one to expect that income is the most important factor, and once that is established as true for the case of Brazil too, there also appear as relevant factors ease of access to health services and sanitation. This evidence leads on to the discussion of alternative nutrition policies, showing the magnitude of the redistribution income necessary to cover the nutritional gap and of the role of the meals and nutrition programs, in their latest form (the so called Social Priorities Program, of the present Government).
\end{abstract}

UNITERMS: Malnutrition. Socioeconomic factors. Income. Population. Child, nutritional status.

\title{
REFERENCIAS BIBLIOGRÁFICAS
}

1. CAMPINO, A.C.C. Nutrição e economia: considerações sobre políticas de curto e médio prazos. São Paulo, 1977. [Tese de livre docência - Faculdade de Economia e Administração da USP].

2. CAMPINO, A.C.C. Pobreza e nutrição. Digesto Econômico (265):223-59, 1979.

3. CAMPINO, A.C.C. Instrumentos de intervenção no sistema de alimentos: sua influência no abastecimento alimentar. [Apresentado na Conferência Prioridades de Pesquisa Aplicada ao Planejamento em Nutrição e Alimentos, Brasília, CNPq, 1981]

4. CAMPINO, A.C.C. \& FARINA, E.M.M.Q. Magnitude da população desnutrida no Brasil e Programa de Prioridades Sociais do Governo Sarney. São Paulo em Perspectiva, outubro, 1985. [no prelo].

5. CAMPINO, A.C.C. et al. Avaliação sócio econômica do Programa de Alimentação do Trabalhador. São Paulo, FIPE/ USP, 1982. [Mimeografado]

* O trabalho pode ser obtido diretamente com
6. CONTADOR, C.R. Benefícios e custos sociais da política de garantia agrícola no Brasil. In: Veiga, A. Ensaios sobre política agrícola brasileira. São Paulo, Secretaria da Agricultura, 1979. p. 163-205.

7. O ESTADO nutricional de crianças de 6 a 60 meses no município de São Paulo: II análise de dados. São Paulo, IMPEP/ IPE, 1975 [Mimeografado]*

8. FUNDAÇÃO GETULIO VARGAS/INSTITUTO BRASILEIRO DE ECONOMIA. Food consumption in Brazil: family budget survey in the early, 1960 s. Jerusalem, Keter Press, 1960.

9. FUNDAÇĀO IBGE. Anuário Estatistico do Brasil: 1980. Rio de Janeiro, 1981.

10. FUNDAÇÃO IBGE. Estudo Nacional da Despesa Familiar - ENDEF: série. Rio de Janeiro, 1977-81.

11. FUNDAÇÃO IBGE/UNICEF. Perfil estatistico de crianças e mães no Brasil. Rio de Janeiro, 1982. 
CAMPINO, A.C.C. Aspectos sócio-econômicos da desnutrição no Brasil. Pev. Saúde públ., S. Paulo, 20:83-101, 1986.

12. FUNDAÇÃO INSTITUTO DE PESQUISAS ECONÔMICAS/INSTITUTO NACIONAL DE ALIMENTAÇÃO E NUTRIÇÃO (FIPE/INAN). Avaliação do Programa de Nutrição - Brasil BIRD. São Paulo, 1982.

13. GONTIJO, V. Padrões regionais de comportamento econômico dos produtos agrícolas do Estado de São Paulo. Belo Horizonte, Centro de Desenvolvimento e Planejamento Regional, 1975.

14. HUTCHINSON, B. Mobilidade e trabalho: um estudo na cidade de São Paulo. Rio de Janeiro, Editora MEC/INEP Centro Brasileiro de Pesquisas, 1960.

15. LEVINSON, F.J. Morinda: an economic analysis of malnutrition among young children in rural India. Cambridge, Mass., Cornell/Massachussets Institut of Tecnology International Nutritron, 1974.

16. MARTINS, I.S. Revisão na análise da situação alimentar no Estado de São Paulo. Rev, ABIA/SAPRO (45):2-8, nov. 1979.
17. PASTORE, A.C. A resposta da produção agrícola aos preços no Brasil. São Paulo, 1968. [Tese de Duutorado - Faculdade de Economia e Administração da USP]

18. SIGULEM, D.M. Contribuição ao estudo da desnutrição energético-protéica, em crianças de 6 a 60 meses no município de São Paulo. São Paulo, 1980. [Dissertação de mestrado - Escola Paulista de Medicina]

19. SIGULEM, D.M. Condições de saúde e nutrição de lactantes no município de São Paulo. São Paulo, 1981. [Tese de doutorado - Escola Paulista de Medicina]

20. SOBRAL, G. Demanda de alimentos no Brasil: arroz, batatinha, carne, feijão e leite - período 50-70. Piracicaba, 1973. [Dissertação de mestrado - Escola Superior de Agricultura "Luiz de Queiroz" da USP]

Recebido para publicação em 13/03/1985. Reapresentado em 13/11/1985.

Aprovado para publicação em 18/11/1985. 